\title{
$-\mathrm{O}$ \\ Global Loss Diversification in the Insurance Sector
}

\author{
Oleg Sheremet ${ }^{1}$ \\ André Lucas² $^{2}$
}

1 Institute for Environmental Studies (IVM), VU University Amsterdam;

2 VU University Amsterdam, Tinbergen Institute, and Duisenberg School of Finance. 


\section{Tinbergen Institute}

The Tinbergen Institute is the institute for economic research of the Erasmus Universiteit Rotterdam, Universiteit van Amsterdam, and Vrije Universiteit Amsterdam.

Tinbergen Institute Amsterdam

Roetersstraat 31

1018 WB Amsterdam

The Netherlands

Tel.: +31(0)205513500

Fax: $+31(0) 205513555$

Tinbergen Institute Rotterdam

Burg. Oudlaan 50

3062 PA Rotterdam

The Netherlands

Tel.: + $31(0) 104088900$

Fax: $+31(0) 104089031$

Most TI discussion papers can be downloaded at http://www.tinbergen.nl. 


\title{
Global Loss Diversification in the Insurance Sector
}

\author{
Oleg Sheremet* and André Lucas ${ }^{\dagger}$
}

\begin{abstract}
We study the possibility for international diversification of catastrophe risk by the insurance sector. Adopting the argument that large insurance losses may be a 'globalizing factor' for the industry, we study the dependence of geographically distant insurance markets via equity returns. In particular, we employ conditional copula theory to model the bivariate dependence of the insurance industry. In contrast to earlier literature on this subject, we disentangle the causes of dependence stemming from the asset side from those from the liability side by conditioning on general market conditions. We find that for both Europe-America and Europe-Asia the dependence is significant. Moreover, we find asymmetric effects: the international dependence is particularly high for losses, even after conditioning for the asset side dependence. Finally, we investigate the time variation in copula parameters and find evidence that dependence in the insurance sector has increased over time, thus reducing the scope for international diversification of large losses in this sector.
\end{abstract}

Keywords: Catastrophic insurance losses; Copula and dependence; Diversification JEL Classification: C32, C52, G15

\section{Introduction}

During the past two decades, increased catastrophic losses have exceeded $\$ 40 \mathrm{bn}$ almost every year, reaching a peak of about $\$ 230$ bn in 2005 , according to SwissRe (2007). Only one third of these losses was insured. Moreover, despite the worldwide increase in reinsurance capacity, the ability of the market to adequately reinsure large catastrophic risks remains questionable, see e.g. Cummins and Weiss (2000) and Froot (2001). In particular, reinsurance coverage is limited and predominantly covers only lower layers of losses, see Froot (2001). It is well-known that it is very difficult to diversify large catastrophic losses locally due to their typical correlation structure. The limited depth of the reinsurance industry, however, seems to suggest that the diversification possibilities for catastrophic losses are also limited on a global scale.

\footnotetext{
*Institute for Environmental Studies (IVM), VU University Amsterdam. E-mail: oleg.sheremet@ivm.vu.nl.

$\dagger$ VU University Amsterdam, Tinbergen Institute, and Duisenberg school of finance. E-mail: alucas@feweb.vu.nl.
} 
In the present paper, we investigate whether the limited reinsurance capacity for catastrophic risks at a global scale is due to an increased international dependence in the insurance industry. We find significant dependence across different world regions. This dependence hints at one of the possible causes of limited capacity of catastrophe reinsurance via traditional reinsurers. Consequently, alternative reinsurance solutions become more important, such as increasing reinsurance capacity directly via capital markets using, for example, catastrophe bonds and securitization.

A theoretical explanation for the limited scale of catastrophe reinsurance markets is given by Froot and O'Connell (2008). They derive that the supply of reinsurance for a given contract is reduced if the variance of losses under that contract is greater (as is the case with catastrophe losses) or the covariance of losses under that contract with the loss distribution of a reinsurer's portfolio is greater (e.g., due to increased cross-sectional dependence of the reinsured catastrophic losses). So, if dependence is strong and has gradually increased globally, reinsurers are less willing to take on new catastrophe insurance contracts, as this would only increase the degree of dependence of their portfolio. Consequently, this limits the scope for global reinsurance.

We empirically determine the dependence between insurance losses using the copula approach for insurers' equity returns. We do so by constructing a dynamic copula model for insurers' equity returns. The usefulness of copula-based methods over traditional correlationbased methods in the financial risk management setting has been worked out in e.g. McNeil et al. (2005). Via copulas we can separate the modeling process for the marginal behavior of losses from that of the dependence structure, as was proved by Sklar (1959). Patton (2006) has extended the copula approach to the dynamic context, such that time variation in the dependence measures is allowed for.

As mentioned, our input to the copula consists of equity returns rather than insurance losses. There are several reasons for this. First, catastrophic losses are typically only available at a very low frequency. This makes it impossible to reliably estimate any dependence measures over different global regions. Second, reported losses may be subject to particular accounting practices. For example, losses may be manipulated for profit smoothing purposes, thus corrupting the dependence structure. Equity returns, by contrast, are available at high frequencies. They constitute the clearest signals of the market's perception of an insurer's position and value at any moment in time.

Naturally, equity returns also have some drawbacks as a proxy for insurance losses. As explained in Slijkerman (2006), the dependence between equity returns in the insurance sector can be the result of exposures to a broad range of similar risks, for example due to correlated losses, but also due to holding similar globally diversified investment portfolios on the asset side of the balance sheet. Slijkerman (2006) measures lower tail dependence among twelve European insurers and reinsurers. He finds that in a bivariate setting insurers demonstrate a higher degree of tail dependence than reinsurers. His conclusion is that the systemic risk in the insurance sector is larger than in the reinsurance sector. In a 
related study, Geluk and De Vries (2006) provide interesting theoretical and empirical results based on this idea of common risk exposures. The authors use results of Extreme Value Theory to prove that unless all reinsurance companies hold non-connected asset and liability portfolios, the reinsurers' equity returns exhibit tail dependence. The conclusion is that while diversification is beneficial for small risks, it may introduce systemic risk for large catastrophic events.

Other related work includes a number of studies on dependence from a contagion perspective. Angbazo and Narayanan (1996) analyze the impact of Hurricane Andrew on the stock prices of 48 publicly traded property-liability insurance firms. The impact was measured as a three-day cumulative abnormal return. Hurricane Andrew negatively affected the majority of firms in their sample. This effect was unrelated to the firms' exposure levels. Fields, Klein, and Myskowski (1998) and Polonchek and Miller (1999) study contagion effects generated by announcements about operational loss events. All of these papers found strong evidence of contagion.

Our paper contributes to the literature in the following ways. First, we investigate the dependence structure between insurance companies' equity returns in three globally disconnected regions (America, Europe, Asia and Australia) to study potential problems for the global reinsurance market brought about by catastrophic losses. Second, in contrast to earlier literature on this subject, we try to disentangle the causes of dependence stemming from the asset side and those from the liability side by conditioning insurers' equity returns on general market conditions. As a measure of dependence, we use both correlation and tail dependence coefficients. Finally, we investigate whether the extremal dependence structure between regions changes over time using the dynamic copula approach of Patton (2006) and the test for possible breakpoints for copula parameters proposed by Dias and Embrechts (2003).

We find that while there are no explicit jumps in dependence between different geographic regions, both tail dependence and correlation show certain variability over time for all market pairs and also have been increasing since about 2004. Also, for all market pairs the dependence of negative returns is higher than the dependence of positive returns. This implies a reduced scope for global diversification of large losses, with the possible exception of America-Asia market, where most of the dependence between equity returns appears to be due to correlated asset rather than liability portfolios.

The paper proceeds as follows. Section 2 describes the methodology and general outline of our model. Section 3 describes the data. Section 4 reports the univariate results. Section 5 then proceeds with the copula models. Finally, Section 6 concludes. 


\section{Model description}

\subsection{Copula theory and dependence modeling}

In this paper we use copulas to model the dependence between the equity returns of insurance companies in different regions. The unconditional bivariate copula is defined as a dependence function of two univariate marginal distributions, such that a joint distribution of two variables may be decomposed into its copula and two marginal distributions:

$$
F_{X Y}(x, y)=C\left(F_{X}(x), F_{Y}(y)\right)
$$

where $C\left(u_{1}, u_{2}\right)$ is a distribution function on the unit square, $\left(u_{1}, u_{2}\right) \in[0,1]^{2}$.

This is the so-called Sklar (1959) representation theorem. The representation in (1) allows us to separate the modeling stage of the marginal distributions $F_{X}(x)$ and $F_{Y}(y)$ from that of the dependence function $C\left(u_{1}, u_{2}\right)$. This latter function is called a copula. As highlighted by McNeil et al. (2005), copulas are a useful extension and generalization of commonly used approaches for modeling joint distributions, such as the correlation concept. In the bivariate setting, copulas allow one to define a range of non-parametric measures of dependence that go beyond the linear correlation coefficient.

For example, an important concept in the context of copulas and dependence in the extreme tails of the distribution is the coefficient of tail dependence. This coefficient is defined as the conditional probability of a large event in one market given a large event in the other market. Mathematically, we define the coefficients of upper $\left(\tau_{U}\right)$ and lower $\left(\tau_{L}\right)$ tail dependence as

$$
\begin{gathered}
\tau_{L}=\lim _{u \rightarrow 0^{+}} P\left(x_{2} \leq F_{2}^{-1}(u) \mid x_{1} \leq F_{1}^{-1}(u)\right)=\lim _{u \rightarrow 0^{+}} \frac{C(u, u)}{u}, \\
\tau_{U}=\lim _{u \rightarrow 1^{-}} P\left(x_{2}>F_{2}^{-1}(u) \mid x_{1}>F_{1}^{-1}(u)\right)=\lim _{u \rightarrow 1^{-}} \frac{\hat{C}(1-u, 1-u)}{1-u}=\lim _{u \rightarrow 0^{+}} \frac{\hat{C}(u, u)}{u},
\end{gathered}
$$

where $\hat{C}(u, v)=C(1-u, 1-v)+u+v-1$ is a survival copula.

Patton (2006) extended the unconditional copula approach to that of a conditional copula,

$$
F_{X Y \mid W}(x, y \mid w)=C\left(F_{X \mid W}(x \mid w), F_{Y \mid W}(y \mid w) \mid w\right)
$$

where $w \in W$ is the conditioning variable. The main advantage of the conditional copula approach is that it allows us to introduce time-varying copulas and thus study changes in dependence structures over time.

In this paper we consider a number of different copulas that are used in the empirical paper of Patton (2006) and can also be found in Denuit et al. (2005), Joe (1997), and Nelsen (1999). In particular, we study the Gaussian, Student $t$, Clayton, Survival Clayton, and Symmetrized Clayton copulas. 
The Gaussian copula is given by

$$
C_{G a}\left(u_{1}, u_{2}\right)=\int_{-\infty}^{\Phi^{-1}\left(u_{1}\right)} \int_{-\infty}^{\Phi^{-1}\left(u_{2}\right)} \frac{1}{2 \pi \sqrt{1-\rho^{2}}} \cdot \exp \left\{-\frac{\zeta_{1}^{2}-2 \rho \zeta_{1} \zeta_{2}+\zeta_{2}^{2}}{2\left(1-\rho^{2}\right)}\right\} d \zeta_{1} d \zeta_{2}
$$

where $\zeta_{i}=\Phi^{-1}\left(u_{i}\right), i=1,2$ is the inverse univariate standard normal distribution function. Dependence for this copula is linear and measured by a single parameter: the coefficient of correlation $\rho$.

As is well known, the Gaussian copula does not permit a non-zero tail dependence coefficient (unless $\rho= \pm 1$ ). A first extension that does allow for non-zero tail dependence is the Student $t$ copula, given by

$$
C_{S t}\left(u_{1}, u_{2}\right)=\int_{-\infty}^{t \cdot \nu^{-1}\left(u_{1}\right)} \int_{-\infty}^{t \cdot \nu^{-1}\left(u_{2}\right)} \frac{1}{2 \pi \sqrt{1-\rho^{2}}} \cdot\left(1+\frac{\xi_{1}^{2}-2 \rho \xi_{1} \xi_{2}+\xi_{2}^{2}}{\nu\left(1-\rho^{2}\right)}\right)^{-\frac{\nu+2}{2}} d \xi_{1} d \xi_{2},
$$

where $\xi_{i}=t_{\nu}^{-1}\left(u_{i}\right), i=1,2$ is the inverse Student $t$ distribution function with $\nu$ degrees of freedom. The tail dependence coefficient equals $\tau=2 t_{\nu+1}\left(-\sqrt{\frac{(\nu+1)(1-\rho)}{1-\rho}}\right)$.

Both the Gaussian and Student $t$ copulas are symmetric. To allow for non-symmetric upper versus lower tail dependence, we consider different versions of the Clayton copula. The Clayton copula is given by

$$
C_{C l}\left(u_{1}, u_{2}\right)=\left(u_{1}^{-a}+u_{2}^{-a}-1\right)^{-\frac{1}{a}}, \quad a \neq 0,
$$

with coefficient of lower tail dependence $\tau_{L}=2^{-1 / a}$, and upper tail dependence $\tau_{U}=0$. Similarly, we consider the survival Clayton copula $\hat{C}(u, v)$ with $\tau_{L}=0$ and $\tau_{U}=2^{-1 / a}$. Finally, we follow Patton (2006) and also consider the symmetrization of this copula,

$$
C^{s y m}\left(u, v \mid \tau_{L}, \tau_{U}\right)=\frac{1}{2} \cdot\left(C\left(u, v \mid \tau_{L}\right)+\hat{C}\left(u, v \mid \tau_{U}\right)\right)
$$

thus allowing for (possibly different) levels of upper and lower tail dependence, simultaneously.

\subsection{Model design and estimation}

As mentioned in the introduction, joint movements in equity returns of insurance companies could be due to reasons unrelated to the insurers' liability side. For example, there could be a general shift in risk premia across markets. Alternatively, insurance companies are likely to hold similar, globally well-diversified investment portfolios. To single out the effects directly related to the liability rather than to the asset side of the balance sheet of insurance firms, we estimate a CAPM regression of the form

$$
r_{i t}-r_{f t}=\alpha_{i}+\beta_{i}\left(r_{m t}-r_{f t}\right)+\varepsilon_{i t},
$$


where $r_{i t}$ is the equity return for insurance firms in a specific geographic region, Europe, North America, and Australasia. The market return $r_{m t}$ is a global market return in our context. In particular, we use the MSCI Global Index return. For the risk-free rate $r_{f}$, we take the return on 3-month US T-bills. The regression in (9) effectively allows us to control for any dependence coming from joint movements of stock markets in different regions. By concentrating on the residuals $\varepsilon_{i t}$ in (9), we hope to filter out most of these effects.

When specifying a model of bivariate dependence between return series using a copula, we actually have to specify three models: the univariate models for the two marginal distributions and the copula model. There are several approaches to fit a copula, see e.g. McNeil et al. (2005). In this paper we employ the so-called two-stage maximum likelihood method (or Inference Functions for Margins or IFM method) with parametric estimators for the marginal distributions at the first stage.

For the margins, we use univariate ARMA-GARCH models with skewed $t$-innovations suggested by Hansen (1994). These models are flexible enough to give an adequate representation of the marginal distribution of equity returns. We fit these models to the market model residuals $\varepsilon_{i t}$ from (9).

The standardized residuals $\hat{z}_{i t}$ from the fitted ARMA-GARCH models are used to generate pseudo-samples $u_{i t}=F\left(\hat{z}_{i t}\right), i=1, \ldots m, t=1, \ldots n$. We use the pseudo-sample for the estimation of the copula parameters and for the selection of the best-fit copula. Under standard conditions, the estimates obtained from the two-stage procedure are consistent and asymptotically normal, though less efficient than under the joint estimation of the parameters of both the univariate margins and the copula. The IFM method is further described in Joe (1997).

For testing the stability of the copula parameters over time, we employ the LR test promoted by Dias and Embrechts (2003). This test checks for change points and their timing in the context of parametric copula models. If there are several change points, the detection is based on the so-called binary segmentation procedure. After a break date has been found, the test procedure is applied to each of the subsamples before and after the break date. For the details of the procedure and the relevant distribution theory, we refer the reader to the above-cited paper and references provided in it.

After the estimation of the copula parameters, the analysis of its goodness-of-fit, and the selection of the most appropriate unconditional copulas, we turn to the analysis of conditional copulas. Following Patton (2006), we assume that the copulas are conditioned on the past values of their parameters and the pseudo-sample. This means that only the copula parameters are varying over time, while the functional form is fixed.

The parametrization of the time variation in the conditional copulas is defined as

$$
\tilde{\rho}_{i l, t}=\omega_{\rho}+\beta_{\rho} \cdot \tilde{\rho}_{i l, t-1}+\alpha_{\rho} \cdot \frac{1}{k} \sum_{j=1}^{k} F^{-1}\left(u_{i, t-j}\right) \cdot F^{-1}\left(u_{l, t-j}\right),
$$

for the correlation coefficient $\rho_{i l, t}=\tilde{\Lambda}\left(\tilde{\rho}_{i l, t}\right)$ in case of the Normal or Student $t$ copulas, and 


$$
\tilde{\tau}_{i l, t}^{L, U}=\omega_{L, U}+\beta_{L, U} \cdot \tilde{\tau}_{i l, t-1}^{U, L}+\alpha_{U, L} \cdot \frac{1}{k} \sum_{j=1}^{k}\left|u_{i, t-j}-u_{l, t-j}\right|,
$$

for tail dependence coefficients $\tau_{i l, t}^{L, U}=\Lambda\left(\tilde{\tau}_{i l, t}^{L, U}\right)$ in case of the copulas that allow for a non-zero tail dependence. Here $\tilde{\Lambda}(x)=\left(1-e^{-x}\right) /\left(1+e^{-x}\right)$ and $\Lambda(x)=\left(1+e^{-x}\right)^{-1}$ are transformations used to keep correlation and tail dependence coefficients in the $(-1,1)$ and $(0,1)$ ranges, respectively. The function $F^{-1}(\cdot)$ is the inverse cumulative distribution function, which is the univariate normal for the Gaussian copula, or the Student $t$ for the Student $t$ copula.

The evolution equations consist of an autoregressive term and a forcing variable. The number of lags $k$ depends on our assumptions on how persistent the variations to dependence are in each particular case. Determining it is an empirical issue. Effectively, the last term in (11) plays the role of a "smoothing" parameter. We consider $k=4 \ldots 26$, which in our case of weekly data is roughly in line with Patton (2006).

\section{Data}

We study the dependence between equity return series for three geographically distinct insurance markets. These return series were formed by aggregating weekly asset returns for publicly traded non-life insurance companies listed in the USA and Canada, Western Europe, and Asia and Australia. For simplicity, we refer to these regions as America, Europe, and Australasia.

Life and health insurance sectors were excluded from consideration. First, not every catastrophe results in large (insured) human life losses, and so property losses represent a more direct impact of a severe loss event on the insurance industry as a whole. Second, as Brewer and Jackson (2002) argue, the life insurance sector differs in operational principles from the non-life sector in that the former has developed closer links with the banking sector. As a result, the life insurance sector may have easier access to external capital and thus be more stable during large catastrophe events. On the other hand, Brewer and Jackson (2002) and later Cummins et al. (2006) show that such a link may bring in additional dependence between equity returns for life insurance companies, and may also channel crises from the banking sector into the insurance sector.

The data come from the Thomson Datastream and the CRSP databases. For the US data, we use CRSP. Our sample comprises equity returns for fire, marine, and casualty insurance companies, which includes a broad range of damages to property. The data for Europe and Australasia are taken from the Datastream and cover a somewhat broader range of the insurance companies. We delete all companies with names that relate to the life insurance industry. This selection was especially important for the Australasian market, where most of the listed insurance companies in the data set have life insurance as their core business. Second, we delete all companies with prolonged non-trading periods. Our 
final sample covers the most liquid publicly traded property insurance firms. The American market has 221 companies (including 73 dead firms), the European market 129 (34 dead), and the Australasian market 98 (11 dead).

For each region, we construct an equally weighted index of log-returns. The sample covers weekly observations from January 1986 to December 2006, which gives us 1095 data points for each series. Table 1 provides summary statistics of the data.

From the reported unconditional moments in the table, we see that all series are negatively skewed. The skeweness parameters are highly significant. This also holds for the kurtosis. Taken together, the empirical distributions of returns display significant non-normal behavior. The test statistics in Table 1 suggest there is significant autocorrelation in the equity return series for all three markets. In addition, heteroscedasticity tests show that all series have significant conditional heteroscedasticity. Both of these empirical stylized facts, together with the excess skewness and kurtosis, are accounted for by the fat-tailed ARMA-GARCH models we use for the marginal distributions.

The correlation matrix in Table 2 shows that there is a substantial positive linear dependence between global insurance market returns. The correlation is strongest between America and Europe, followed by Europe-Australasia and America-Australasia.

To get a first impression of the degree of tail dependence, we compute the exceedance correlations. The exceedance correlation is defined as the correlation between exceedance returns, that is between $\left\{r_{1}: r_{1} \geq F_{1}^{-1}(\theta)\right\}$ and $\left\{r_{2}: r_{2} \geq F_{2}^{-1}(\theta)\right\}$ for $\theta \geq 1 / 2$, and between $\left\{r_{1}: r_{1} \leq F_{1}^{-1}(\theta)\right\}$ and $\left\{r_{2}: r_{2} \leq F_{2}^{-1}(\theta)\right\}$ for $\theta \leq 1 / 2$, where $F_{i}^{-1}(\theta)$ denotes the $\theta$-quantile of the empirical distribution of $r_{i}$. More details can be found in Longin and Solnik (2001). These plots, reshaped as a difference between exceedance correlations for the original returns and exceedance correlations for artificial series generated from the bivariate normal distribution with the same correlation coefficient as the empirical returns, are displayed in the left panel of the Figure 1. Looking at the graphs, we see substantial deviations from bivariate normality. If the returns had been generated from the normal distribution, both positive and negative exceedance correlation differences would have been close to zero for both lower and upper tails. This is not the case for our data. We can see substantial correlation of both large negative and positive return exceedances for EuropeAmerica, Europe-Australasia and America-Australasia. However, the large positive returns in all three market pairs tend to be less correlated than the large negative returns. Moreover, for positive returns, the exceedance correlations in some cases even change sign and become negative.

\section{Univariate modeling}

\subsection{Accounting for asset-side dependence}

In order to exclude possible assets-side dependence between the equity returns for insurance companies operating on geographically distant markets, we need to filter out joint equity 
movements brought about by general movements in worldwide securities markets. To do so, we fit an international CAPM to each of our return series. Table 3 reports the results. For all regions, the results are comparable. The constant terms are insignificantly different from zero, and the $\beta$ S are statistically significant and of similar size. The international CAPM therefore cannot be rejected in this case.

The correlations of the original equity returns and those of the CAPM residuals are compared in the bottom line of Table 2. As expected, the asset-side dependence indeed contributes significantly to the overall dependence of the returns. For Europe-America and Europe-Australasia, the correlation between the CAPM residuals is about $40 \%$ lower than between the original returns. For America-Australasia the effect is even more pronounced, as the correlation falls by $78 \%$. The fact that the results of insurance activities at the American and the Australasian markets are almost uncorrelated implies there may be substantial scope for diversification of insurance losses across these markets. Also, after controling for asset side dependence, the correlation between the Europe-America and Europe-Australasia markets is very moderate $(0.20-0.25)$, though statistically significant. The correlation, however, only captures the linear dependence between the different markets. We are also interested in other forms of dependence, particularly in the tails of the distribution. In the next section we investigate these more general dependencies via copula modeling.

\subsection{Models for marginals}

Before we can specify the copulas, we have to establish the marginal models for each market. Preliminary specification tests revealed that the best models for the marginal distributions are $\operatorname{AR}(2)-\operatorname{GARCH}(1,1)$ for Europe, $\operatorname{ARMA}(2,1)-\operatorname{GARCH}(1,1)$ for America, and $\operatorname{ARMA}(1,1)-\operatorname{GARCH}(2,2)$ for Australasia. To account for the leptokurtic nature of the data as found in Table 1, we choose the skewed Student $t$ distribution of Hansen (1994) as the distribution for innovations in our univariate models. Parameter estimation is performed by maximum likelihood using Ox and G@ARCH, see Doornik (2006) and Laurent and Peters (2006). The estimation results are available upon request.

After the univariate modeling stage, the pattern of tail dependence of the residuals has changed considerably. The right-hand panel in Figure 1 presents the differences between exceedance correlations of the residuals obtained after the CAPM and ARMA-GARCH filtering and the corresponding bivariate normal random values. Accounting for the asset side co-movements, skewness, kurtosis, autocorrelation, and volatility clustering results in reduced correlations of negative exceedances. In the far left tail, however, high exceedance correlations are still observed, though these estimates are subject to a larger sampling error. The largest losses thus show a much higher degree of extreme co-movement than implied by the normal distribution. The higher clustering for larger losses between the markets is worrying from a diversification perspective.

In the right-hand tail, by contrast, tail behavior appears to be much closer to that of the bivariate normal. Still, the Europe-America pair shows a switch in correlations in the 
right-hand tail. Whereas large losses tend to co-move in these regions, gains tend to offset each other. This is precisely the reverse of what one would hope global diversification would accomplish. The same, though to a lesser extent, holds for the pair Europe-Australasia. Finally, the America-Australasia combination reveals higher clustering of both large losses and large gains in these markets. Again, the former is worrysome, while the latter may be advantageous in this case.

To conclude, we gather from the exceedance correlation plots that loss dependence in the insurance sector may show a lack of diversification for large loss sizes. The increased exceedance correlations show up between all pairs of globally distinct regions and may limit the scope of re-insurance or catastrophe risk swaps. We now try to capture these dependencies among large losses via the copula approach and try to study more formally whether there is extreme loss dependence, and if so, whether this dependence is stable over time.

\section{Bivariate dependence models}

\subsection{Unconditional copulas}

We start with the determination of the unconditional copulas, i.e., the static copulas with time-invariant parameters. Following Dias and Embrechts (2003), we consider a number of different copulas, namely the Gaussian, Student $t$, Clayton, Survival Clayton, and Symmetrized Clayton copula as defined in (5)-(8). We thus consider copulas that allow for tail dependence or tail independence, either in the right or left tail of the return distribution, or in both. The estimates of the copula parameters for the complete sample 1986-2006 are presented in Table 4.

Table 4 reveals that for the Gaussian copula the correlation parameter between Europe and Australasia and between Europe and America are very close, 0.25 and 0.22 , respectively. As was suggested by the preliminary analysis, the correlation between America and Australasia is much lower (0.07 only). As the Gaussian copula does not allow for asymptotic tail dependence, we turn to the Student $t$ copula. The estimates of tail dependence for the Student $t$ copula are, however, not significantly different from zero for any market pair. Both the Gaussian and the Student $t$ copula yield similar estimates of the correlation between the filtered returns. Both the Gaussian and Student $t$ copula, however, are symmetric. To allow for asymmetry in the tail dependence as suggested by the preliminary data analysis, we turn to the Clayton copula.

Estimates of the tail dependence parameters for the Clayton and Survival Clayton copulas support our earlier preliminary results about asymmetry in the tail dependence for Europe-America and Europe-Australasia. For both market pairs the lower tail dependence coefficients are statistically significant and higher than the upper tail dependence. The estimated degree of tail dependence is modest over globally distant regions, the probability of a joint crash or catastrophic loss in two regions given a large loss in one region always remains below 10\%. For America-Australasia, the estimates of the tail dependence parameters are 
not statistically significant. In terms of fit, the Student $t$ and Symmetrized Clayton seem to outperform the other copulas for all markets.

\subsection{Stability of copula parameters}

As one of the elements in this paper is to investigate the time stability of the global dependence between insurance losses, we also present as a first check the estimation results for the different copulas over two subsamples, January 1986 - June 1996 and July 1996 December 2006, in Table 4. The only significant difference appears for the Europe-America pair. Closer inspection reveals that this is mainly due to increased clustering in the lower tail, i.e., increased clustering of large losses. Also the upper-tail dependence increases over the two subsamples, but not in a statistically significant way.

The Table 4, however, only presents the results for a very specific choice of break date. To allow for an endogenous choice of the break date and general possible shifts in the dependence parameters of the unconditional copulas, we apply LR test proposed by Dias and Embrechts (2003). The test computes the LR statistic for all possible break dates. The supremum of all resulting values over all break dates is taken as the test statistic. Dias and Embrechts provide the statistical theory for this supremum test. The test can be repeatedly applied over subsamples in order to detect multiple breaks. A brief description of the test is provided in the appendix.

Figure 2 illustrates the test statistics for the lower tail dependence parameter of the Clayton copula fitted to all three market pairs over the entire 1986-2006 period. The figure graphs the value of the likelihood ratio versus the break date. The horizontal line indicates the critical value of the Dias-Embrechts test. It is clear from the figure that for the Clayton copula no significant (endogenous) breaks in dependence can be detected for Europe-America. The figure also shows that the maximum difference in dependence is reached roughly halfway the sample, as evidenced by the earlier results in Table 4.

There is a single break in dependence (significant at $5 \%$ level) for Europe-Australasia towards the end of the sample period, in April 2005. Again we see that the evidence for a structural change in dependence mainly accumulates during the second half of the sample. For America-Australasia, there is a significant break roughly halfway the sample, though somewhat later than the split implemented in Table 4. The results are similar for the other copulas, the major difference being that we detect a second, earlier break in 1992 for America-Australasia using the Student $t$ copula.

We can compare the break dates with the available data on major catastrophic events in each global region. The list of events is presented in Table 6 in the appendix and is compiled from the annual reports published by Swiss Re, e.g., SwissRe (2007). The events are denoted as symbols for all regions at the bottom of each panel in Figure 2. Some tentative relations can be established based on these specific events. For Europe-America, there is a clear increase in the number of joint events in the second half of the sample. This appears in line with the earlier estimation results. We can interpret this as increased dependence caused 
by both accumulating catastrophic losses from a chain of major catastrophic events and spreading market apprehension of increased catastrophic hazards worldwide. For EuropeAustralasia, the picture is less clear. There appears to be a higher incidence of joint events at the start and at the end of the sample, with relatively less (joint) activity in the middle. An illustration is the change in the dependence for Europe-Australasia that becomes most pronounced after several hurricanes and a tsunami in Australasia in September-December 2004, followed by the winter storm in Europe in January 2005. For America-Australasia, the list of catastrohpes provides no clear signal that the incidence of joint events has increased or decreased over time and therefore casts some doubt on the formal statistical test results and their economic significance.

The mixed results on time variation so far can be explained in several ways. First, the data might provide little information on changes in the extreme dependence structure given the span of the sample period. Second, the Dias-Embrechts test might lack power to detect smaller changes, for example if they occur very gradually over time. Finally, it may be the case that there is genuine time variation rather than a structural change in the dependence structure. To investigate this in more detail, we turn to the estimation of our time-varying conditional copula models.

\subsection{Conditional copulas}

Drawing on the above results for the fixed copulas, we now concentrate our analysis on the dynamics of the dependence measures. We limit the reported results to the Gaussian and Symmetrized Clayton conditional copulas only. The estimation results for conditional copulas are reported in Table 5 and illustrated by Figure 3. The graphs of the conditional copula parameters are again complemented by symbols indicating the dates of major natural catastrophes.

Though the specification of conditional copulas is straightforward, their estimation is much less so. Our analysis revealed that the estimation results for the conditional copulas are sensistive to specifications of parameters in (10)-(11), as well as to starting values. The number of lags $k$, which is effectively a smoothing parameter, influences the convergence of the numerical likelihood maximization procedure. After some preliminary experiments, we selected $k=20$ for yielding the most stable estimation results, thus implicitly assuming averaging for the dependence dynamics over events within a half year time frame. We used various starting values to minimize the risk of ending up in a local rather than a grobal maximum of the likelihood.

For the Gaussian copulas, Table 5 reveals a high degree of persistence. This is evidenced by the high values of $\beta$ and by the patterns in the top panels of Figure 3 .

For Europe-America, the time variation of both the correlation and the lower tail dependence measure agrees well with our conclusion on the basis of LR tests. The increase in dependence was relatively gradual, with several temporary upturns during 1987-mid-1991, 1994-1997, and 2000-2004. The upper tail dependence parameter, by contrast, remained 
relatively stable on average during the whole 1986-2006 period, though with considerable short-term noise. In particular, the upper tail dependence coefficient exhibits spikes at the beginning of the periods of upturns in the lower tail dependence coefficient, but returns to normal levels afterwards.

Comparing the dates of high dependence with the available data on natural catastrophes in Table 6 shows that the periods of increased dependence for Europe-America are associated with the consecutive occurrence of several catastrophic events. For example, the dependence reaches a local peak after America was hit by several hurricanes in October-November 1989 followed by winter storms hitting Europe in December-February 1990. The next increase in dependence in 2000-2004 started approximately after the hurricane season in America in September 1999 followed by winter storms in Europe in December 1999. This period covered several catastrophes that occurred closely in time. We also observe higher dependence at the time when several major catastrophic events occur closely in time on one market, as was the case during 1995-1997 in America. Generalizing these observations, we can say that during the periods of several consecutive catastrophes in both regions or in the immediate aftermath of a series of large catastrophe events on a single market the geographically distant insurance markets of Europe and America tended to display a higher degree of comovement.

For Europe-Australasia, the dynamics of the correlation and lower tail dependence parameters are quite similar. There are two large periods of higher dependence, in particular during 1989-1995 and 2000-2006. As for Europe-America, local peaks of dependence seem to coincide with major catastrophes occurring closely in time in both regions or with a cluster of catastrophic losses on one of the markets. There is also a period of reduced dependence during 1996-1999, when there were no major catastrophic events in either region. On the other side, the upper tail dependence coefficient exhibits considerable short-term volatility without any clear long-term upward or downward trend. Still, the periods of deviation from the mean dependence are to a certain degree visible even in the chaotic dynamics of the upper tail dependence parameter.

For America-Australasia, results on the time dynamics of the different dependence measures are not easily reconciled. On one hand, the time-varying correlation parameter displays a strong upward trend in the second half of the sample. This is clearly in line with the split sample estimation results for the static copulas in Table 4. The results for the Symmetrized Clayton copula, however, reveal a different picture. The estimation results, however, are highly sensitive to small changes in the specification or starting values. This is in line with the preliminary analyses and the results for the static copulas: the America-Australasia pair shows so little clustering of catastrophic events that the data contain hardly any information on the dependence structure, whether static or dynamic. This is further supported by the list of catastrophic events as indicated by the symbols at the bottom of each panel.

This leads us to our overall conclusion that the scope for global diversification in the insurance sector very much depends on the regions over which one diversifies. Australasia and America appear to offer the largest potential gains from bilateral diversification. Also 
the other regions still have scope for loss diversification. However, one of the other signals emerging from our analysis is that the dependence structure between these regions may be changing over time. In particular, there is some mild evidence that dependence increases over time. Though the time span of our sample is too short to draw any firm conclusions in this respect, it is interesting to also consider climatic change as one of the possible causes for this phenomenon. This would require the reinsurance market to become increasingly cautious in exploiting global diversification as a means for risk reduction.

\section{Conclusion}

In this paper we analyzed possibilities for global diversification of large insurance risks, looking at occurrences of loss clustering and investigating the degree of comovement of the equity returns on non-life insurance markets in Europe, America, and Australasia. Assuming that large losses may be a 'globalizing factor' for the insurance industry, we investigate whether the increase in economic and insured losses observed during the last two decades is associated with increased dependence of the geographically distant insurance markets.

We found that for Europe-America and Europe-Australasia, the liability-side dependence accounts for about $60 \%$ of the total observed dependence of the aggregated equity return series for insurance markets, while about $40 \%$ is due to dependence resulting from the stock market comovements (that is, due to asset-side dependence). However, for AmericaAustralasia the assets-side dependence accounts for as much as almost $80 \%$ of the total observed dependence between aggregate equity returns for the non-life insurance companies.

Thus, even after controlling for asset-side comovements, the degree of dependence resulting from insurance and reinsurance operations is still significantly different from zero. We found that Europe-America and Europe-Australasia insurance markets exhibit relatively similar degree of dependence estimated over the whole period 1986-2006. Moreover, the analysis shows that the dependence is asymmetric. The estimation reveals that lower tail dependence is higher than the upper tail dependence: clustering of large losses is more prevalent than that of gains.

The dependence parameters are not fixed over the entire period under consideration. Several of our tests indicate that the correlation and tail dependence parameters of the unconditional copulas fitted for Europe-Australasia and America-Australasia have breakpoints or display significant time-variation.

No single catastrophe in the past two decades seems to be linked to an abrupt shift in the dependence of equity returns for insurance companies in Europe-America, EuropeAustralasia, or America-Australasia. Rather, our analysis of the dynamics of the dependence parameters in the conditional copula framework shows that changes in the dependence are gradual and associated with a series of natural catastrophes.

To summarize, large insurance losses indeed play the role of the 'globalizing' factor, especially for Europe and America. This means that the scope for international diversification of 
catastrophic losses is limited. In particular, the most severely affected reinsurance schemes may be retrocession or catastrophe risk swaps. In addition, the increased dependence of geographically distant insurance markets may undermine portfolio diversification benefits of catastrophe bonds, because different issues of such bonds may be subject to increasingly strong dependence, thus limiting their cross-regional diversification benefits.

As a possible way to cope with these unfavorable developments, we suggest to look at diversification possibilities in Australasia, which in our analysis revealed the least codependence with the other markets.

\section{A LR test for detection of change points}

The test below is due to Dias and Embrechts (2003).

Let $u_{1}, u_{2}, \ldots u_{n}$ be a sequence of independent random vectors in $[0,1]^{d}$ with univariate uniformly distributed margins and copulas $C\left(u ; \theta_{1}\right), \ldots C\left(u ; \theta_{n}\right)$ respectively, where $\theta_{i}$ are the copula parameters. Formally, we test the null hypothesis

$$
H_{0}: \theta_{1}=\ldots=\theta_{n}
$$

versus the alternative

$$
H_{1}: \theta_{1}=\theta_{k} \neq \theta_{k+1}=\ldots=\theta_{n} .
$$

If we reject the null hypothesis, $k$ is the time of the change. All the parameters of the model are assumed to be unknown under both hypotheses. If $k$ were known, the null hypothesis would be rejected for small values of the likelihood ratio

$$
\Lambda_{k}=\frac{\sup \prod_{1 \leq i \leq n} c\left(u_{i} ; \theta\right)}{\sup \prod_{1 \leq i \leq k} c\left(u_{i} ; \theta\right) \prod_{k<i \leq n} c\left(u_{i} ; \theta^{\prime}\right)}
$$

where the estimation of $\Lambda_{k}$ is carried out through ML and so all necessary conditions of regularity and efficiency have to be assumed.

Denote

$$
\begin{aligned}
L_{k}(\theta) & =\sum_{1 \leq i \leq k} \log c\left(u_{i} ; \theta\right), \\
L_{k}^{*}(\theta *) & =\sum_{k<i \leq n} \log c\left(u_{i} ; \theta\right) .
\end{aligned}
$$

Then the likelihood ratio can be written as

$$
-2 \cdot \log \left(\Lambda_{k}\right)=2\left(L_{k}(\hat{\theta})+L_{k}^{*}\left(\hat{\theta^{*}}\right)-L_{n}(\hat{\theta})\right),
$$


and $H_{0}$ will be rejected for large values of

$$
Z_{n}=\max _{1 \leq i \leq n}\left(-2 \cdot \log \left(\Lambda_{k}\right)\right)
$$

The following approximation holds for large critical values and small samples:

$$
\begin{gathered}
P\left(Z_{n}^{1 / 2} \geq x\right) \approx \frac{x^{p} \exp \left(-x^{2} / 2\right)}{2^{p / 2} \Gamma(p / 2)} \times \\
\times\left(\log \frac{(1-h)(1-l)}{h l}-\frac{p}{x^{2}} \cdot \log \frac{(1-h)(1-l)}{h l}+\frac{4}{x^{2}}+O\left(\frac{1}{x^{4}}\right)\right),
\end{gathered}
$$

where $h$ and $l$ can be taken as $h(n)=l(n)=(\log n)^{3 / 2} / n$, and $p$ is the number of parameters that may change under the alternative.

\section{B List of catastrophes in $1996-2006$}

Catastrophic events selected for the Table 6 are taken from Sigma annual reports by SwissRe, see, e.g., SwissRe (2007). The lower limit for losses to be included in the table was set at USD $700 \mathrm{mn}$.

\section{References}

Angbazo, L. A. and R. Narayanan (1996). Catastrophic shocks in the property-liability insurance industry: evidence on regulatory and contagion effects. The Journal of Risk and Insurance 63(4), 619-637.

Brewer, E. I. and W. E. I. Jackson (2002). Inter-industry contagion and the competitive effects of financial distress announcements: Evidence from commercial banks and life insurance companies. Federal Reserve Bank of Chikago Working Papers.

Cummins, D. J. and M. Weiss (2000). The global market for reinsurance: Consolidation, capacity, and efficiency. Brookings-Wharton Papers on Financial Services.

Cummins, J. D., R. Wei, and C. M. Lewis (2006, October). The market value impact of operational loss events for us banks and insurers. Journal of Banking \& Finance 30(10), 2605-2634.

Denuit, M., J. Dhaene, M. Goovaerts, and R. Kaas (2005). Actuarial Theory for Dependent Risks. Measures, Orders, and Models. John Wiley \& Sons.

Dias, A. and P. Embrechts (2003). New Risk Measures in Investment and Regulation, Chapter Change-point analysis for dependence structures in finance and insurance, pp. 69-86. John Wiley \& Sons. 
Doornik, J. A. (2006). Ox 4: An Object Oriented Matrix Programming Language. Timberlake Consultant Ltd.

Fields, J. A., L. S. Klein, and E. G. Myskowski (1998). Lloyd's financial distress and contagion within the us property and liability insurance. Journal of Risk and Uncertainty 16(2), 173-185.

Froot, K. and P. O'Connell (2008). On the pricing of intermediate risk: Theory and application to catastrophe reinsurance. Journal of Banking and Finance 32, 69-85.

Froot, K. A. (2001). The market for catastrophe risk: a clinical examination. Journal of Financial Economics 60, 529-571.

Geluk, J. and C. De Vries (2006). Weighted sums of subexponential random variables and asymptotic dependence between returns on reinsurance equities. Insurance: Mathematics and Economics 38, 39-56.

Hansen, B. (1994). Autoregressive conditional density estimation. International Economic Review 35 (3), 705-730.

Joe, H. (1997). Multivariate Models and Dependence Concepts. Chapman \& Hall/CRC.

Laurent, S. and J.-P. Peters (2006). G@RCH 4.2 : An Ox package for estimating and forecasting various ARCH models. Timberlake Consultant Ltd.

Longin, F. and B. Solnik (2001). Extreme correlation of international equity markets. The Journal of Finance LVI (2), 649-676.

McNeil, A., R. Frey, and P. Embrechts (2005). Quantitative Risk Management: Concepts, Techniques, and Tools. Princeton University Press.

Nelsen, R. B. (1999). An Introduction to Copulas. New York: Springer-Verlag.

Patton, A. J. (2006). Modeling asymmetric exchange rate dependence. International Economic Review 47 (2), 527-555.

Polonchek, J. and R. K. Miller (1999). Contagion effects in the insurance industry. The Journal of Risk and Insurance 66(3), 459-475.

Sklar, A. (1959). Fonctions de repartition a n dimensions et leurs marges. Publications de l'Institut Statistique de l'Universite de Paris 8, 229-231.

Slijkerman, J. (2006). Insurance sector risk. Tinbergen Institute Discussion Paper 062/2.

SwissRe (2007). Natural catastrophes and man-made disasters (annual reviews). Technical report, SwissRe. 

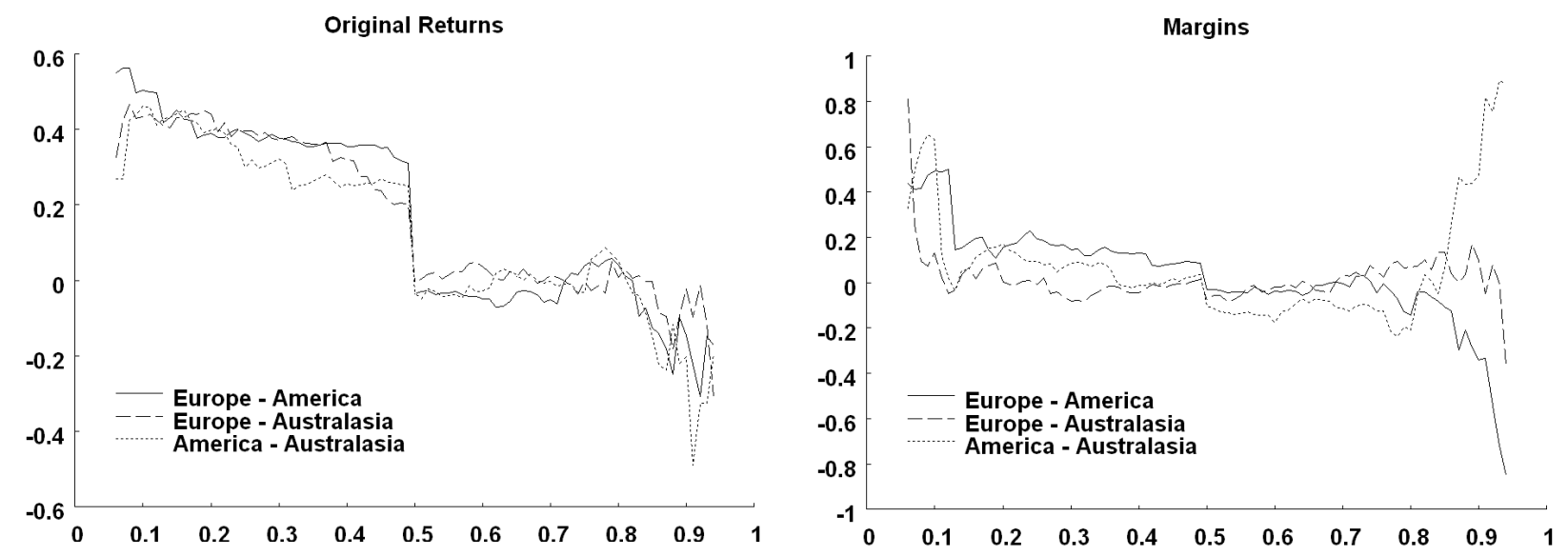

Figure 1: Graphs illustrating tail dependence of return series

The left-hand panel contains differences between the exceedance correlations of the original returns for insurance companies in Europe-America, America-Australasia, and Europe-Australasia, and the exceedance correlations of the bivariate normal random values with the same correlation coefficient as in the empirical data. So for normally distributed returns, the line should be flat at zero. The right-hand panel plots differences between the exceedance correlations of standardized residuals after fitting both CAPM and ARMA-GARCH marginal models, and the exceedance correlations of their bivariate normal counterparts. The horizontal axis is scaled in terms of quantiles of the return series, which means that 0.1 corresponds to large negative excess returns, and 0.9 corresponds to large positive excess returns.
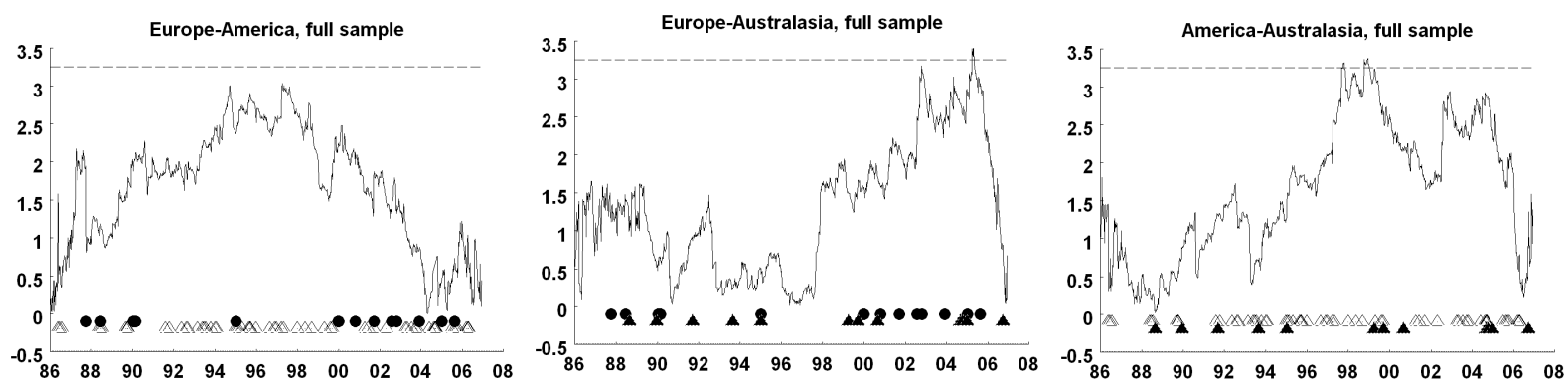

Figure 2: Critical statistic for LR test of parameter stability for unconditional copulas

The graphs present the test statistics for the LR break test for the Clayton copula parameter, see Dias and Embrechts (2003). The left, middle, and right-hand panel correspond to Europe-America, EuropeAustralasia, and America-Australasia, respectively. At the bottom of each panel, major catastrophic events (insured losses exceeding USD 700mn) are displayed. Discs correspond to catastrophic events in Europe, white triangles to events in America, and dark triangles to events in Australasia. The horizontal line indicates the critical value of the test at the $5 \%$ level. 

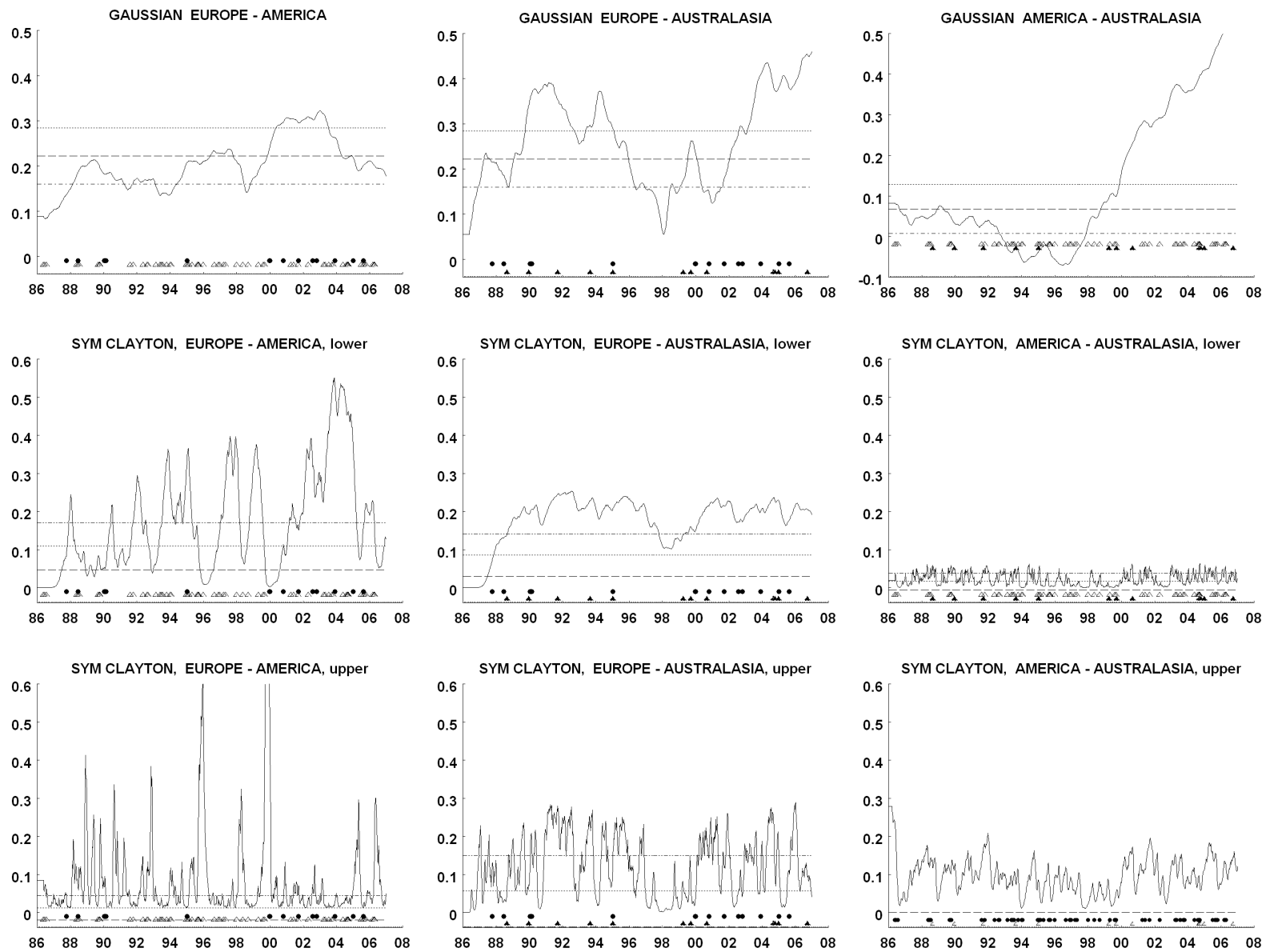

Figure 3: Graphs of dependence parameters for conditional copulas

The graphs present time-varying correlations (upper panel) and lower and upper tail dependence parameters (middle and lower panel) for conditional Gaussian and Symmetrized Clayton copulas estimated for EuropeAmerica (left), Europe-Australasia (center), and America-Australasia (right). Horizontal lines indicate estimates of the corresponding fixed copula parameters and their $95 \%$ level confidence bands. The bottom panel of each graph shows dates of major catastrophic events for corresponding regions. Only catastrophe events with insured losses exceeding USD 700mn were selected. Dots correspond to catastrophic events in Europe, white triangles to events in America, and dark triangles to events in Australasia. 
Table 1: Summary statistics for aggregated weekly return series for non-life insurance markets in Europe, America, and Australasia

Data on weekly equally-weighted equity log-return indices for the non-life insurance sector over January 1986 to December 2006 come from the Thomson Datastream and the CRSP database. All returns are in US dollar terms. JB stat means the Jarque-Bera normality test. Q-stat (10) is the Ljung-Box Q-statistics for autocorrelation. Q-stat $r^{2}(10)$ means the Ljung-Box test for conditional heteroscedasticity, applied to squared residuals (10 lags). All individual and test statistics are significant at $1 \%$ level.

\begin{tabular}{rccc}
\hline \multicolumn{4}{c}{ Statistics } \\
\hline \hline Europe & America & Australasia \\
\hline min & $-12.1^{* *}$ & $-13.9^{* *}$ & $-13.5^{* *}$ \\
std.dev & $7.7^{* *}$ & $7.3^{* *}$ & $13.5^{* *}$ \\
skewness & $-0.1^{* *}$ & $1.7^{* *}$ & $2.6^{* *}$ \\
kurtosis & $6.5^{* *}$ & $-0.9^{* *}$ & $-0.1^{* *}$ \\
\hline JB stat & $703.3^{* *}$ & $2723.0^{* *}$ & $6.5^{* *}$ \\
Q-stat $(10)$ & $51.6^{* *}$ & $32.6^{* *}$ & $133.4^{* *}$ \\
Q-stat $r^{2}(10)$ & $102.9^{* *}$ & $179.0^{* *}$ & $933.9^{* *}$ \\
\hline
\end{tabular}

Table 2: Correlation coefficients for aggregated weekly returns and CAPM residuals

Correlations between the raw returns are in the top panel. Correlations between residuals after fitting the international CAPM are in the bottom panel, together with the percentage changes compared to the correlations in raw returns. $* *$ indicates significance at the $1 \%$ level.

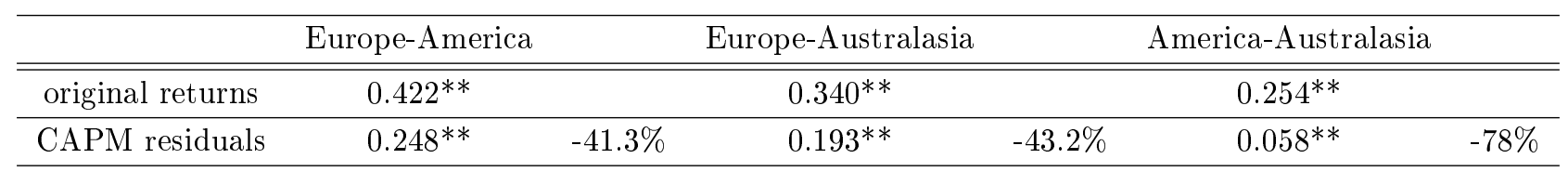

Table 3: CAPM estimates for aggregated weekly returns

The model is $r_{i t}-r_{f t}=\alpha+\beta_{i}\left(r_{m t}-r_{f t}\right)+\varepsilon_{i t}$, where $r_{i t}$ is an insurance sector return for each of the geographic markets under consideration (Europe, America, and Australasia), $r_{m t}$ is the MSCI Global Index return, and $r_{f t}$ is the return on three-month US T-bills. Standard errors are given in parentheses.

Estimates marked $* *$ are significant at the $1 \%$ level.

\begin{tabular}{|c|c|c|c|c|c|c|}
\hline Estimation & \multicolumn{2}{|c|}{ Europe } & \multicolumn{2}{|c|}{ America } & \multicolumn{2}{|c|}{ Australasia } \\
\hline const & 0.00046 & $(0.001)$ & -0.00035 & $(0.000)$ & 0.00074 & $(0.001)$ \\
\hline$\beta$ & $0.459^{* *}$ & (0.027) & $0.420^{* *}$ & (0.021) & $0.519^{* *}$ & $(0.035)$ \\
\hline $\operatorname{Adj.} R^{2}$ & 0.20 & & 0.27 & & 0.17 & \\
\hline
\end{tabular}


Table 4: Parameter estimates for bivariate unconditional copulas

The table presents parameter estimates for all five bivariate unconditional (fixed) copulas. Standard errors of the estimates are given in parentheses. Loglikelihood values are reported in brackets. ${ }^{*}$ and ${ }^{* *}$ indicate significance at the $5 \%$ and $1 \%$ level, respectively. Applied to LogL, $*$ and $* *$ indicate that the copula parameters are significantly different over subsamples based on the likelihood ratio test statistic provided in the last column.

\begin{tabular}{cccccccc}
\hline \multicolumn{7}{c}{ Europe - America } \\
\hline \hline \multirow{2}{*}{ Gaussian } & $\rho^{G a}$ & $0.222^{* *}$ & $(0.031)$ & $0.156^{* *}$ & $(0.041)$ & $0.285^{* *}$ & $(0.037)$ \\
& LogL & {$[27.8]$} & & {$[6.7]^{*}$} & & {$[23.8]^{*}$} & 5.4 \\
\hline \multirow{2}{*}{ Student $t$} & $\rho^{S t}$ & $0.220^{* *}$ & $(0.029)$ & $0.150^{* *}$ & $(0.045)$ & $0.286^{* *}$ & $(0.044)$ \\
& $\nu^{S t}$ & $15.1^{*}$ & $(7.5)$ & 10.35 & $(4.96)$ & 36.5 & $(56.3)$ \\
& $\tau^{S t}$ & 0.0054 & $(0.010)$ & 0.014 & $(0.022)$ & 0.0 & $(0.0)$ \\
& $\operatorname{LogL}$ & {$[30.3]$} & & {$[9.3]^{*}$} & & {$[24.1]^{*}$} & 6.2 \\
\hline Clayton & $\tau_{L}^{C}$ & $0.066^{* *}$ & $(0.029)$ & 0.013 & $(0.018)$ & $0.153^{* *}$ & $(0.049)$ \\
& $\operatorname{LogL}$ & {$[26.4]$} & & {$[6.8]^{*}$} & & {$[22.9]^{*}$} & 6.6 \\
\hline Surv.Clayton & $\tau_{U}^{\hat{C}}$ & $0.042^{*}$ & $(0.025)$ & 0.015 & $(0.022)$ & 0.076 & $(0.042)$ \\
& $\operatorname{LogL}$ & {$[17.5]$} & & {$[5.0]$} & & {$[13.4]$} & 1.8 \\
\hline \multirow{2}{*}{ Sym.Clayton } & $\tau_{L}^{S C}$ & $0.109^{* *}$ & $(0.031)$ & 0.027 & $(0.041)$ & 0.172 & $(0.096)$ \\
& $\tau_{U}^{S C}$ & 0.012 & $(0.016)$ & 0.014 & $(0.031)$ & 0.018 & $(0.026)$ \\
& $\operatorname{LogL}$ & {$[30.3]$} & & {$[8.4]^{*}$} & & {$[24.6]^{*}$} & 5.4 \\
\hline \hline
\end{tabular}

Europe - Australasia

\begin{tabular}{cccccccc}
\hline & \multicolumn{3}{c}{ full sample } & \multicolumn{2}{c}{ first half } & \multicolumn{2}{c}{ second half } \\
\hline \hline Gaussian & $\rho^{G a}$ & $0.249^{* *}$ & $(0.027)$ & $0.239^{* *}$ & $(0.038)$ & $0.261^{* *}$ & $(0.039)$ \\
& $\operatorname{LogL}$ & {$[35.4]$} & & {$[17.3]$} & & {$[18.3]$} & 0.4 \\
\hline \multirow{2}{*}{ Student $t$} & $\rho^{S t}$ & $0.250^{* *}$ & $(0.029)$ & 0.250 & $(0.144)$ & $0.264^{* *}$ & $(0.041)$ \\
& $\nu^{S t}$ & $71.5^{* *}$ & $(2.4)$ & 27.6 & $(2.75)$ & 192 & $(15.0)$ \\
& $\tau^{S t}$ & 0.0 & $(0.0)$ & 0.0003 & $(0.0001)$ & 0.0 & $(0.0)$ \\
& $\operatorname{LogL}$ & {$[35.5]$} & & {$[17.7]$} & & {$[18.3]$} & 1.0 \\
\hline Clayton & $\tau_{L}^{C}$ & $0.082^{* *}$ & $(0.031)$ & $0.087^{*}$ & $(0.044)$ & 0.076 & $(0.043)$ \\
& $\operatorname{LogL}$ & {$[28.6]$} & & {$[16]$} & & {$[12.7]$} & 0.4 \\
\hline Surv.Clayton & $\tau_{U}^{C}$ & $0.066^{*}$ & $(0.030)$ & 0.045 & $(0.035)$ & $0.094^{*}$ & $(0.049)$ \\
& $\log \mathrm{L}$ & {$[22.0]$} & & {$[9.8]$} & & {$[12.6]$} & 0.8 \\
\hline Sym.Clayton & $\tau_{L}^{S C}$ & $0.085^{* *}$ & $(0.028)$ & 0.127 & $(0.092)$ & 0.046 & $(0.055)$ \\
& $\tau_{U}^{S C}$ & 0.056 & $(0.047)$ & 0.019 & $(0.031)$ & 0.106 & $(0.096)$ \\
& $\log \mathrm{L}$ & {$[33.6]$} & & {$[18.4]$} & & {$[16]$} & 1.6 \\
\hline \hline
\end{tabular}

America - Australasia

\begin{tabular}{cccccccc}
\hline & \multicolumn{3}{c}{ full sample } & \multicolumn{2}{c}{ first half } & \multicolumn{2}{c}{ second half } \\
\hline \hline Gaussian & $\rho^{G a}$ & $0.068^{*}$ & $(0.030)$ & 0.014 & $(0.043)$ & $0.119^{*}$ & $(0.041)$ \\
& $\operatorname{LogL}$ & {$[2.53]$} & & {$[0.05]$} & & {$[4.0]$} & 3.04 \\
\hline \multirow{2}{*}{ Student $t$} & $\rho^{S t}$ & $0.069^{*}$ & $(0.030)$ & 0.012 & $(0.045)$ & $0.121^{*}$ & $(0.044)$ \\
& $\nu^{S t}$ & 30.6 & $(27.8)$ & 19.8 & $(16.6)$ & 56.7 & $(139)$ \\
& $\tau^{S t}$ & 0.0 & $(0.0)$ & 0.0002 & $(0.001)$ & 0.0 & $(0.0)$ \\
& $\operatorname{LogL}$ & {$[3.2]$} & & {$[0.85]$} & & {$[4.1]$} & 3.5 \\
\hline Clayton & $\tau_{L}^{C}$ & 0.0005 & $(0.001)$ & 0.0 & $(0.0)$ & 0.007 & $(0.012)$ \\
& $\operatorname{LogL}$ & {$[4.5]$} & & {$[0.72]$} & & {$[4.7]$} & 1.84 \\
\hline \multirow{2}{*}{ Surv.Clayton } & $\tau_{U}^{C}$ & 0.0 & $(0.0)$ & - & - & 0.0002 & $(0.001)$ \\
& $\log \mathrm{L}$ & {$[0.3]$} & & - & & {$[1.0]$} & \\
\hline \multirow{2}{*}{ Sym.Clayton } & $\tau_{L}^{S C}$ & 0.016 & $(0.011)$ & 0.0004 & $(0.001)$ & 0.044 & $(1.0)$ \\
& $\tau_{U}^{S C}$ & 0.0 & $(0.0)$ & 0.0 & $(0.0)$ & 0.0 & $(0.0)$ \\
& $\log \mathrm{L}$ & {$[4.7]$} & & $21^{0.73]}$ & & {$[4.7]$} & 1.46 \\
\hline
\end{tabular}


Table 5: Estimates of time-varying dependence parameters of conditional copulas

The table reports parameter estimates of the conditional copula models in (10-11). Standard errors are reported in parentheses. ${ }^{*}$ and ${ }^{* *}$ indicate significance at the $5 \%$ and $1 \%$ level, respectively.

\begin{tabular}{ccccccc}
\hline & Europe - America & \multicolumn{3}{c}{ Europe - Australasia } & \multicolumn{3}{c}{ America - Australasia } \\
\hline \multicolumn{7}{c}{ Gaussian } \\
\hline$\omega$ & 0.012 & $(0.008)$ & $-0.021^{*}$ & $(0.008)$ & 0.015 & $(0.165)$ \\
$\alpha$ & -0.046 & $(0.033)$ & $0.104^{*}$ & $(0.037)$ & -0.061 & $(0.376)$ \\
$\beta$ & $1.0005^{* *}$ & $(0.003)$ & $0.990^{* *}$ & $(0.006)$ & $1.005^{* *}$ & $(0.002)$ \\
$\operatorname{LogL}$ & {$[31.41]$} & \multicolumn{5}{c}{$[8.36]$} \\
\hline \multicolumn{7}{c}{ Sym. Clayton } \\
\hline$\omega^{L}$ & 0.062 & $(0.042)$ & -0.035 & $(0.080)$ & -1.711 & $(2.814)$ \\
$\alpha^{L}$ & -0.086 & $(0.052)$ & -0.013 & $(0.033)$ & -0.632 & $(1.436)$ \\
$\beta^{L}$ & $0.970^{* *}$ & $(0.025)$ & $0.962^{* *}$ & $(0.071)$ & 0.357 & $(0.669)$ \\
\hline$\omega^{U}$ & -6.020 & $(3.337)$ & -0.190 & $(0.615)$ & -4.952 & $(9.487)$ \\
$\alpha^{U}$ & -1.813 & $(1.180)$ & -0.285 & $(0.396)$ & 1.210 & $(2.482)$ \\
$\beta^{U}$ & 0.476 & $(0.278)$ & 0.734 & $(0.486)$ & $0.945^{* *}$ & $(0.039)$ \\
$\log \mathrm{L}$ & {$[35.09]$} & & {$[35.78]$} & & {$[5.39]$} & \\
\hline
\end{tabular}


Table 6: Catastrophic events during 1986-2006

\begin{tabular}{|c|c|c|c|c|c|}
\hline \multirow[t]{2}{*}{ Date } & \multirow[t]{2}{*}{ Type } & \multirow[t]{2}{*}{ Loss, $\$ m l n$} & \multicolumn{3}{|c|}{ Location: } \\
\hline & & & Europe & America & Asia \\
\hline Summer 1986 & Drought & 1500 & & $\mathrm{x}$ & \\
\hline October 15,1987 & Autumn storm & 4230 & $\mathrm{x}$ & & \\
\hline Summer 1988 & Drought & 4000 & & $\mathrm{x}$ & \\
\hline July 6,1988 & Explosion platform Piper Alpha & 2712 & $\mathrm{x}$ & & \\
\hline September 12, 1988 & Cyclone Gilbert & 1509 & & & $\mathrm{x}$ \\
\hline September 15, 1989 & Hurricane Hugo & 5427 & & $\mathrm{x}$ & \\
\hline October 17,1989 & Earthquake Loma Prieta & 1294 & & $\mathrm{x}$ & \\
\hline October 23,1989 & Explosion Phillips Petroleum & 1714 & & $\mathrm{x}$ & \\
\hline December 28, 1989 & Earthquake Newcastle & 902 & & & $\mathrm{x}$ \\
\hline January 25, 1990 & Winter storm Daria & 5636 & $\mathrm{x}$ & & \\
\hline February 3, 1990 & Storm Herta & 1022 & $\mathrm{x}$ & & \\
\hline February 26, 1990 & Winter storm Vivian & 3917 & $\mathrm{x}$ & & \\
\hline February 28, 1990 & Winter storm Wiebke & 936 & $\mathrm{x}$ & & \\
\hline August 18, 1991 & Hurricane Bob & 989 & & $\mathrm{x}$ & \\
\hline September 27, 1991 & Hurricane Mireille & 6542 & & & $\mathrm{x}$ \\
\hline October 20, 1991 & Forest fire & 1413 & & $\mathrm{x}$ & \\
\hline August 24, 1992 & Hurricane Andrew & 18286 & & $\mathrm{x}$ & \\
\hline September 11, 1992 & Hurricane Iniki & 1829 & & $\mathrm{x}$ & \\
\hline March 10, 1993 & Blizzard over East coast & 1943 & & $\mathrm{x}$ & \\
\hline Summer 1993 & Floods & 21000 & & $\mathrm{x}$ & \\
\hline September 3, 1993 & Typhoon Yancy & 995 & & & $\mathrm{x}$ \\
\hline October 26, 1993 & Forest fire & 1055 & & $\mathrm{x}$ & \\
\hline January 17, 1994 & Northridge earthquake & 13529 & & $\mathrm{x}$ & \\
\hline February x, 1994 & Winter storm & 3000 & & $\mathrm{x}$ & \\
\hline January 17, 1995 & Earthquake Kobe & 2603 & & & $\mathrm{x}$ \\
\hline January 21, 1995 & Storms, floods & 1053 & $\mathrm{x}$ & & \\
\hline January-March 1995 & Floods & 3000 & & $\mathrm{x}$ & \\
\hline May 5, 1995 & Wind, hail, floods & 1195 & & $\mathrm{x}$ & \\
\hline September 3, 1995 & Hurricane Luis & 1579 & & $\mathrm{x}$ & \\
\hline September 14, 1995 & Hurricane Marilyn & 921 & & $\mathrm{x}$ & \\
\hline October 4,1995 & Hurricane Opal & 2211 & & $\mathrm{x}$ & \\
\hline January x, 1996 & Blizzard, floods & 3000 & & $\mathrm{x}$ & \\
\hline September 5, 1996 & Hurricane Fran & 1637 & & $\mathrm{x}$ & \\
\hline December 1996-January 1997 & Torrential floods & 3000 & & $\mathrm{x}$ & \\
\hline April-May 1997 & Floods & 3700 & & $\mathrm{x}$ & \\
\hline January 5, 1998 & Cold spell & 1231 & & $\mathrm{x}$ & \\
\hline May 15, 1998 & Wind, hail, floods & 1345 & & $\mathrm{x}$ & \\
\hline
\end{tabular}




\begin{tabular}{|c|c|c|c|c|c|}
\hline Date & Type & Loss, $\$ \mathrm{mln}$ & & Location: & \\
\hline & & & Europe & America & Asia \\
\hline September 20, 1998 & Hurricane Georges & 3530 & & $\mathrm{x}$ & \\
\hline April 14, 1999 & Hailstorm Sydney & 982 & & & $\mathrm{x}$ \\
\hline May 3, 1999 & Tornadoes & 1485 & & $\mathrm{x}$ & \\
\hline August 17, 1999 & Earthquake in Turkey - Izmit & 2000 & & & $\mathrm{x}$ \\
\hline September 10, 1999 & Hurricane Floyd & 2360 & & $\mathrm{x}$ & \\
\hline September 20, 1999 & Earthquake Nantou & 1000 & & & $\mathrm{x}$ \\
\hline September 22, 1999 & Typhoon Bart & 2980 & & & $\mathrm{x}$ \\
\hline December 25, 1999 & Winter storm Lotar & 4500 & $\mathrm{x}$ & & \\
\hline December 27, 1999 & Winter storm Martin & 2200 & $\mathrm{x}$ & & \\
\hline September 10, 2000 & Tokai floods & 990 & & & $\mathrm{x}$ \\
\hline October 29,2000 & Floods after Storm Oratia & 747 & $\mathrm{x}$ & & \\
\hline April 6, 2001 & Hailstorms, floods & 1900 & & $\mathrm{x}$ & \\
\hline June 5, 2001 & Storm Allison & 3150 & & $\mathrm{x}$ & \\
\hline September 11, 2001 & Terrorist attack on WTC & 19000 & & $\mathrm{x}$ & \\
\hline September 21, 2001 & Explosion in France & 1357 & $\mathrm{x}$ & & \\
\hline April 27, 2002 & Several tornadoes & 1675 & & $\mathrm{x}$ & \\
\hline July 31,2002 & Flooding & 700 & $\mathrm{x}$ & & \\
\hline August 6, 2002 & Flooding & 2500 & $\mathrm{x}$ & & \\
\hline October 26,2002 & Storm Jeanet & 845 & $\mathrm{x}$ & & \\
\hline April 4, 2003 & Hailstorms, floods & 1605 & & $\mathrm{x}$ & \\
\hline May 2, 2003 & Tornadoes & 3205 & & $\mathrm{x}$ & \\
\hline July 21, 2003 & Hail & 815 & & $\mathrm{x}$ & \\
\hline September 18, 2003 & Hurricane Isabel & 1685 & & $\mathrm{x}$ & \\
\hline October 21, 2003 & Wildfire & 975 & & $\mathrm{x}$ & \\
\hline October 25, 2003 & Cedar fire wildfire & 1060 & & $\mathrm{x}$ & \\
\hline December x, 2003 & Floods & 1009 & $\mathrm{x}$ & & \\
\hline May 21, 2004 & Tornadoes, hail & 805 & & $\mathrm{x}$ & \\
\hline August 11, 2004 & Hurricane Charley & 8000 & & $\mathrm{x}$ & \\
\hline August 26, 2004 & Hurricane Frances & 5000 & & $\mathrm{x}$ & \\
\hline August 30,2004 & Typhoon Chaba & 956 & & & $\mathrm{x}$ \\
\hline September 2, 2004 & Hurricane Ivan & 11000 & & $\mathrm{x}$ & \\
\hline September 6, 2004 & Typhoon Songda & 3585 & & & $\mathrm{x}$ \\
\hline September 13, 2004 & Hurricane Jeanne & 4000 & & $\mathrm{x}$ & \\
\hline October 13, 2004 & Typhoon Tokage & 1119 & & & $\mathrm{x}$ \\
\hline December 26, 2004 & Tsunami & 5000 & & & $\mathrm{x}$ \\
\hline January 8, 2005 & Winter storm Erwin & 1887 & $\mathrm{x}$ & & \\
\hline July 6, 2005 & Hurricane Dennis & 1115 & & $\mathrm{x}$ & \\
\hline August 19, 2005 & Floods & 1864 & $\mathrm{x}$ & & \\
\hline
\end{tabular}




\begin{tabular}{|c|c|c|c|c|c|}
\hline Date & Type & Loss, $\$ \mathrm{mln}$ & & Location: & \\
\hline & & & Europe & America & Asia \\
\hline August 24, 2005 & Hurricane Katrina & 45000 & & $\mathrm{x}$ & \\
\hline September 20, 2005 & Hurricane Rita & 10000 & & $\mathrm{x}$ & \\
\hline October 16,2005 & Hurricane Wilma & 10000 & & $\mathrm{x}$ & \\
\hline March 11, 2006 & Tornadoes & 920 & & $\mathrm{x}$ & \\
\hline April 6, 2006 & Tornadoes, hail & 1282 & & $\mathrm{x}$ & \\
\hline April 13, 2006 & Tornadoes, hail & 1850 & & $\mathrm{x}$ & \\
\hline September 15, 2006 & Typhoon Shanshan & 1024 & & & $\mathrm{x}$ \\
\hline
\end{tabular}

